\title{
The Effectiveness Colonized System of Ganoderma Vaccine Compared to Trichoderma on Oil Palm Plantations That Infected by Ganoderma boninense
}

\author{
Roderick Bastian ${ }^{1}$, Supeno Surija ${ }^{1} \&$ Michelle Faustine ${ }^{1}$ \\ ${ }^{1}$ Plantation Key Technology Research Centre, Medan, Sumatera Utara, Indonesia \\ Correspondence: Supeno Surija, Plantation Key Techonology Research Centre, Medan, Sumatera Utara, \\ Indonesia. Tel: 62-811-615-525. E-mail: supenosurija@pkt-group.com
}

Received: April 28, 2021

Accepted: June 2, $2021 \quad$ Online Published: June 15, 2021

doi:10.5539/jas.v13n7p89

URL: https://doi.org/10.5539/jas.v13n7p89

\begin{abstract}
Basal stem rot caused by Ganoderma boninense causes fall and death of oil palm crops, and will drastically reduce plantation productivity by up to $50 \%$ and become one of the most serious problems in oil palm cultivation. This study aims to determine the effectiveness of the Colonized System of Ganoderma Vaccine (CHIPS $®$ ), as a biocontrol agent against the suppression of Ganoderma disease that infects oil palm trees in-field conditions. This research was conducted from early 2016 to the end of 2020 in a plantation located in Langkat area, North Sumatra by using 216 trees on 1 block as a sample experiment. Plants that infected with Ganoderma is treated with $\mathrm{T} 1$ treatment $\left(\mathrm{MOAF}^{\mathrm{TM}}\right.$ \& $\left.\mathrm{CHIPS}{ }^{\circledR}\right)$ shows the value of Disease Severity Index (DSI) decreasing to $37.50 \%$, compare to T2 treatment (NPK Granular Fertilizer 13-6-27 \& Trichoderma bio fungicides) which has DSI value of $100 \%$ in week 232 (in 2020) and C1 treatment (control) with the highest DSI value of $100 \%$ in week 212 (in 2020). The conclusions of this study shows that the treatment of T1 (MOAFTM \& CHIPS ${ }^{\circledR}$ ) is more successful in eradicating Ganoderma and have the highest profit ratio than any other treatments. The results of this study are useful as references for in-field inspection and large-scale production.
\end{abstract}

Keywords: basal stem rot, Ganoderma boninense, oil palm, Ganoderma Vaccine, CHIPS ${ }^{\circledR}$, Trichoderma

\section{Introduction}

Ganoderma boninense is an eukaryotic organism classified into groups of fungi. Ganoderma gets food heterotrophically by taking food from organic compounds on its surrounding. These organic compounds will be converted into simple molecules and absorbed directly by hyphae. Ganoderma boninense is a saprophyte (can live on plant remains) and will turn pathogenic when living nearby to the roots of oil palm plants (Boulord et al., 2017; Jing, 2007; Paterson, 2007).

Oil palm plantations play an important role in life, such as food sources, raw material sources to global industries, and provide job opportunities. At a time when Indonesia is facing an economic crisis, the palm oil industry is one of the mainstays of agro-industries that hold foreign exchange for the country (Susanto et al., 2013). However, oil palm plantations have many problems with pests and diseases such as basal stem rot caused by Ganoderma boninense (Mohd \& Faridah, 2008).

The negative impact of Ganoderma caused the fall and death of oil palm crops, thus drastically reducing plantation productivity (Sundram et al., 2008). This causes most palm oil businesses to expand their oil palm plantation (extension) which can damage the environment (Liza et al., 2018; Priwiratama \& Susanto, 2014). One of the efforts to increase oil palm tree productivity without damaging the environment is by maximizing oil palm production on existing plantations itself (Cooper et al., 2021; Darmono et al., 2014).

This is based on in-depth research through careful observation, producing a solution, resulting in new findings of Colonized System of Ganoderma Vaccine (CHIPS $\left.{ }^{\circledR}\right)$. This fungi vaccine is high technology generation and environmentally friendly so that basal stem Rot (BSR) disease can be controlled and the economic deterioration of oil palm plantations in Indonesia can be prevented. The invention of CHIPS ${ }^{\circledR}$ is the first step to reduce conversion of forest and peatland for extension of oil palm plantation. 
CHIPS $®$ is a new technology of organic formulations that is a combination of several types and strains of microorganisms such as Trichoderma and other types with an estimated amount of $6 \times 10^{7}$ to $2 \times 10^{8} \mathrm{CFU}$. These strains of microorganisms are prepared with the Colonized Complex System, where these microorganisms will form colonies equipped with logistics so that they can work together to prevent and kill pathogens. These symbioses can finally control Ganoderma Boninense, where all strains of microorganisms in CHIPS ${ }^{\circ}$ can function effectively in-field and not only in the laboratory.

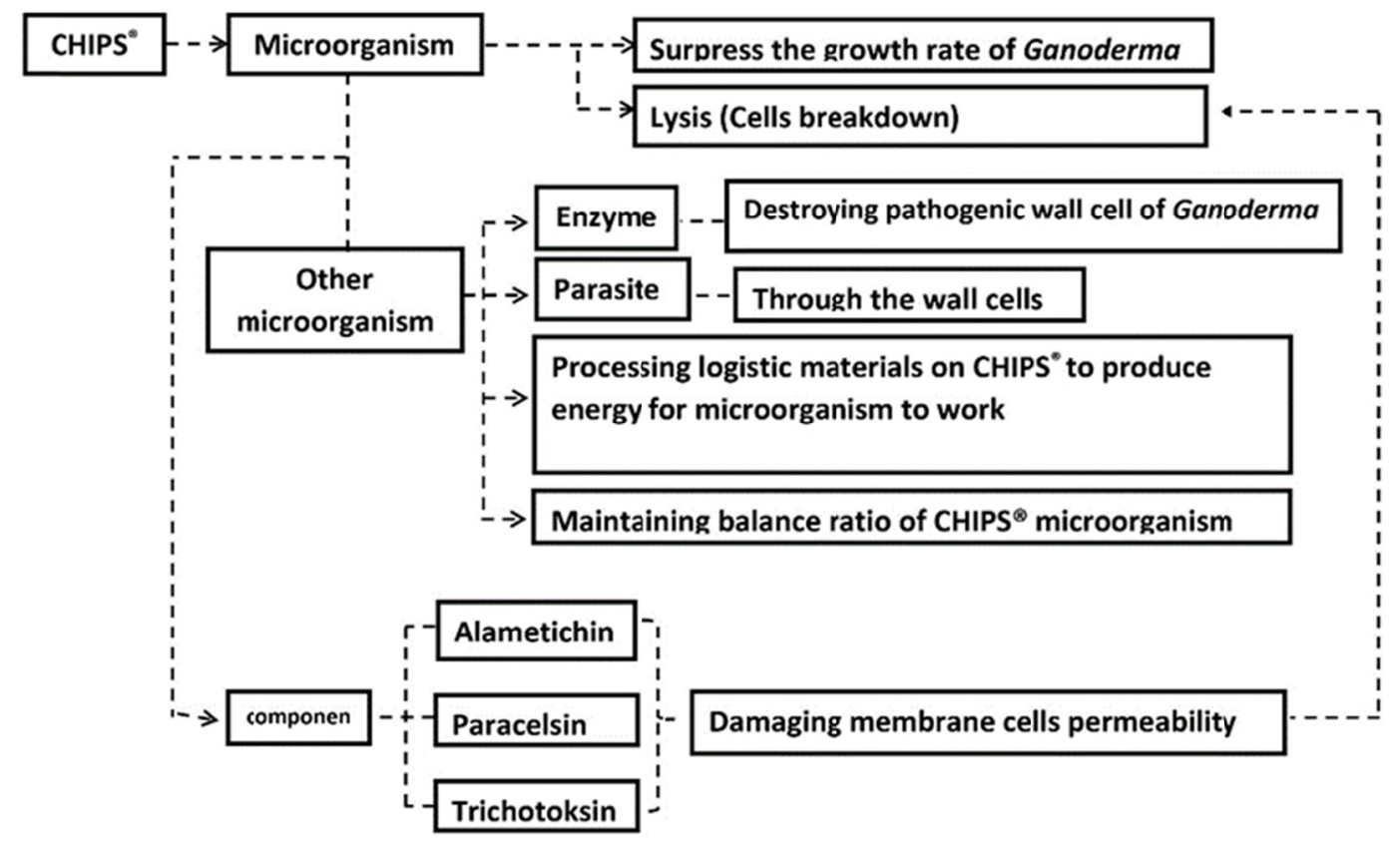

Figure 1. How CHIPS $®$ works

Source: PT PKT.

Collaboration of various microorganisms in CHIPS ${ }^{\circledR}$ will turn fresh Basidiocarp Ganoderma boninense rotten and destroyed.

\section{Materials and Methods}

This research was conducted in early 2016 until the end of 2020 in oil palm plantations located in Langkat area, North Sumatra with an area of $30 \mathrm{Ha}$ (3,906 trees) Division II Block E8, Planting Year of 2006 with mineral soil types. The materials used are 216 tree samples, plant data, MOAF fertilizer ${ }^{\mathrm{TM}}$, CHIPS ${ }^{2}$, NPK Granular Fertilizer 13-6-27 \& Trichoderma Bio fungicides, color paint (red, yellow, green and blue). Tools including stationery, worksite maps, hoes, rake, buckets, jerry cans 25 liters, 5 pieces of plastic burlap, gloves, masks, AP boot shoes, scales and measuring tools of $1 \mathrm{~kg}$ and 60 grams, plastic plates, rustled knives and sickle blade. 


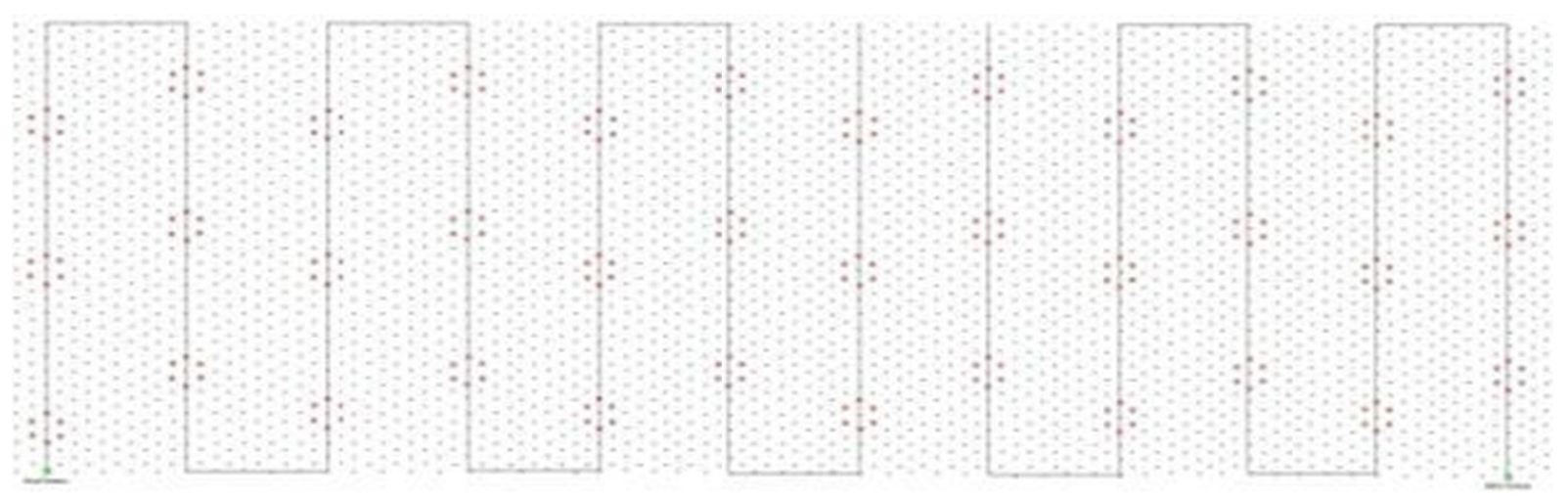

Figure 2. Map of determination of census point

\subsection{Census Point Determination}

Before the selection of samples is done, first we must do a census for point determination. The census points in this study were 36 census points out of an area of $30 \mathrm{Ha}$ (3,960 trees). The census point map can be seen in Figure 2.

\subsection{Sample Selection}

From 216 tree samples there are 4 stages (Stage I, II, III, and IV), for determination on each stage can be seen from the symptoms in plants (Table 1). Stage I has 120 samples, stage II has 48 samples, stage III has 30 samples, and stage IV has 18 samples.

Table 1. Palm oil symptoms in each stage according from PT PKT

\begin{tabular}{ll}
\hline Stage & Symptom \\
\hline O & Healthy plants without the appearance of Ganoderma mycelium on plants. \\
I & Yellowish and dull leaves. The canopy surface is flat and mostly male inflorescence appear. \\
II & $\begin{array}{l}\text { Two spear leaves did not open. Mycelium appears on the midrib basal stem. Fresh fruit bunch was not } \\
\text { ripened but already fell from its spikelet. }\end{array}$ \\
III & $\begin{array}{l}\text { The lower midrib dries up (young plants) and breaks off (old plants), } 3 \text { spear leaves did not open. The } \\
\text { presence of basidiocarp Ganoderma and productivity decrease. } \\
\text { IV }\end{array}$ \\
All leaves break off and dry up, the remaining hang on its trees. The tissue inside of the trees is heavily \\
damaged and trees easily fall off.
\end{tabular}

\subsection{Research Plan}

In this study, sample treatments are divided into 3 treatments, $\mathrm{C} 1, \mathrm{~T} 1$ and $\mathrm{T} 2 . \mathrm{C} 1$ treatment (control) is an infected and uncontrolled sample, T1 treatment is applied with MOAFTM and CHIPS ${ }^{\circledR}$, as well as T2 treatment is applied with NPK Granular fertilizer 13-6-27 and Trichoderma bio fungicides. The research design can be seen in Table 2.

Table 2. Crops sample treatment according infection stages

\begin{tabular}{lllll}
\hline \multirow{2}{*}{ Corps Condition } & \multicolumn{3}{c}{ Treatment } & \multirow{2}{*}{ Total } \\
\cline { 2 - 4 } & $\mathbf{C 1}$ & $\mathbf{T 1}$ & $\mathbf{T 2}$ & 120 trees \\
Stage I & 40 trees & 40 trees & 40 trees & 48 trees \\
Stage II & 16 trees & 16 trees & 16 trees & 30 trees \\
Stage III & 10 trees & 10 trees & 10 trees & 18 trees \\
Stage IV & 6 trees & 6 trees & 6 trees & \\
\hline Total & & & 216 trees \\
\hline
\end{tabular}




\subsection{Treatment}

In $\mathrm{C} 1$ treatment as a control where the samples only use NPK Granular fertilizer 13-627.

- NPK Granular fertilizer 13-6-27 application:

(1) Fertilizer sprinkled on the crop discs with a dose of $2.5 \mathrm{~kg} / \mathrm{tree} / 4$ month.

In $\mathrm{T} 1$ treatment, eradication is carried out using $\mathrm{MOAF}^{\mathrm{TM}}$ as fertilizer and $\mathrm{CHIPS}^{\circledR}$ as Ganoderma biocontrol.

- MOAFTM application:

(1) MOAFTM fertilizer is sprinkled on crop discs with a dose of $3 \mathrm{~kg} /$ tree $/ 6$ month which aims to increase crop yield of palm oil fruit.

- CHIPS ${ }^{\circledR}$ Application:

(1) After 10 days of MOAFTM fertilization, continued with CHIPS ${ }^{\circledR}$ application.

(2) CHIPS ${ }^{\circledR}$ applied on the crop discs with a dose of $3 \mathrm{~kg} /$ tree and re-application after 2 years and 6 months.

T2 treatment using NPK Granular fertilizer 13-6-27 and bio fungicides Trichoderma.

- NPK Granular 13-6-27 application:

(1) Fertilizer sprinkled on crop discs with a dose of $2.5 \mathrm{~kg} / \mathrm{tree} / 4$ month.

- Trichoderma bio fungicides Application:

(1) After 10 days of NPK Granular 13-6-27 fertilization, then continue with application of Trichoderma bio fungicides.

(2) Trichoderma bio fungicides is sprinkled on crop discss with a dose of $400 \mathrm{gr} / \mathrm{tree} / 6$ month.

\subsection{Disease Severity Index (DSI)}

Plants diagnosed with disease on scale 0 to IV stages (Table 1). Each stage of disease is determined for each control treatment samples (C1) and treatment samples (T1 and T2), Disease Severity Index is calculated as follows below.

DSI values were analyzed using non-parametric techniques (Friedman test) SPSS version 19.0. DSI is calculated in every 4 weeks for 5 year based on the following formula:

$$
\text { Disease Severity Index }(\mathrm{DSI})=\frac{\sum(\mathrm{A} \times \mathrm{B}) \times 100}{\Sigma \mathrm{B} \times 4}
$$

where, A: Stage (0, I, II, III or IV); B: Number of plants at each stage/control treatment.

\section{Results}

From the observation, result of Colonized System of Ganoderma Vaccine (CHIPS $\left.{ }^{\circledR}\right)$ compared to Trichoderma bio fungicides on trees that got infected with Ganoderma boninense in 2016-2020 on oil palm plantation located in Langkat area, North Sumatra can be seen in Table 3. 
Table 3. Severity index in every 4 weeks

\begin{tabular}{|c|c|c|c|c|c|c|c|c|c|c|c|c|c|}
\hline \multirow{2}{*}{ Treatment } & \multicolumn{13}{|c|}{2016 (Weeks) } \\
\hline & 4 & 8 & 12 & 16 & 20 & 24 & 28 & 32 & 36 & 40 & 44 & 48 & 52 \\
\hline C1 (without treatment) & 43.75 & 43.75 & 44.79 & 46.88 & 47.57 & 50.00 & 51.39 & 52.08 & 53.47 & 54.17 & 54.86 & 56.25 & 57.29 \\
\hline T1 $\left(\right.$ MOAFTM $^{T}$ CHIPS $\left.^{\circledR}\right)$ & 43.75 & 43.75 & 43.40 & 43.40 & 43.40 & 43.06 & 43.06 & 43.06 & 43.06 & 42.71 & 42.71 & 42.71 & 42.36 \\
\hline $\begin{array}{l}\text { T2 (NPK Granular 13-6-27 \& } \\
\text { Trichoderma Bio fungicides) }\end{array}$ & 43.75 & 43.75 & 44.10 & 44.79 & 46.18 & 47.57 & 47.92 & 49.31 & 50.69 & 51.74 & 53.13 & 53.82 & 54.51 \\
\hline \multirow{2}{*}{ Treatment } & \multicolumn{13}{|c|}{2017 (Weeks) } \\
\hline & 56 & 60 & 64 & 68 & 72 & 76 & 80 & 84 & 88 & 92 & 96 & 100 & 104 \\
\hline C1 (without treatment) & 57.99 & 59.38 & 60.42 & 60.76 & 62.15 & 63.19 & 64.93 & 65.63 & 68.75 & 69.44 & 70.83 & 71.88 & 73.61 \\
\hline T1 $\left(\right.$ MOAFTM $\left.^{\mathrm{T}} \mathrm{CHIPS}^{\circledR}\right)$ & 42.36 & 42.36 & 42.36 & 42.36 & 42.01 & 42.01 & 42.01 & 41.67 & 41.67 & 41.32 & 41.32 & 41.32 & 41.32 \\
\hline $\begin{array}{l}\text { T2 (NPK Granular 13-6-27 \& } \\
\text { Trichoderma Bio fungicides) }\end{array}$ & 54.86 & 55.56 & 56.60 & 57.64 & 57.99 & 58.68 & 60.42 & 62.15 & 63.19 & 64.24 & 65.63 & 66.67 & 68.75 \\
\hline \multirow{2}{*}{ Treatment } & \multicolumn{13}{|c|}{2018 (Weeks) } \\
\hline & 108 & 112 & 116 & 120 & 124 & 128 & 132 & 136 & 140 & 144 & 148 & 152 & 156 \\
\hline C1 (without treatment) & 74.65 & 75.69 & 77.08 & 79.51 & 80.56 & 82.64 & 84.72 & 86.81 & 87.50 & 88.54 & 89.58 & 91.32 & 92.36 \\
\hline T1 (MOAFTM \& CHIPS $\left.{ }^{\circledR}\right)$ & 40.97 & 40.97 & 40.63 & 40.28 & 40.28 & 40.28 & 39.93 & 39.93 & 40.63 & 40.63 & 39.93 & 40.63 & 40.63 \\
\hline $\begin{array}{l}\text { T2 (NPK Granular 13-6-27 \& } \\
\text { Trichoderma Bio fungicides) }\end{array}$ & 70.49 & 72.22 & 73.61 & 76.04 & 77.08 & 77.78 & 79.51 & 80.90 & 82.29 & 83.33 & 84.38 & 85.07 & 85.42 \\
\hline \multirow{2}{*}{ Treatment } & \multicolumn{13}{|c|}{2019 (Weeks) } \\
\hline & 160 & 164 & 168 & 172 & 176 & 180 & 184 & 188 & 192 & 196 & 200 & 204 & 208 \\
\hline C1 (without treatment) & 92.36 & 92.36 & 92.71 & 92.71 & 93.75 & 93.75 & 94.10 & 94.10 & 95.14 & 97.22 & 98.61 & 98.96 & 99.65 \\
\hline T1 $\left(\right.$ MOAFTM $\&$ CHIPS $^{\text {T) }}$ & 40.63 & 40.63 & 40.28 & 40.28 & 40.28 & 39.58 & 39.58 & 39.58 & 39.24 & 39.24 & 39.24 & 39.24 & 39.24 \\
\hline $\begin{array}{l}\text { T2 (NPK Granular 13-6-27 \& } \\
\text { Trichoderma Bio fungicides) }\end{array}$ & 86.11 & 87.15 & 87.85 & 89.58 & 90.97 & 91.32 & 92.01 & 92.71 & 93.75 & 93.75 & 94.10 & 94.44 & 95.49 \\
\hline \multirow{2}{*}{ Treatment } & \multicolumn{13}{|c|}{2020 (Weeks) } \\
\hline & 212 & 216 & 220 & 224 & 228 & 232 & 236 & 240 & 244 & 248 & 252 & 256 & 260 \\
\hline C1 (without treatment) & 100 & 100 & 100 & 100 & 100 & 100 & 100 & 100 & 100 & 100 & 100 & 100 & 100 \\
\hline T1 $\left(\right.$ MOAFTM $\&$ CHIPS $\left.^{\circledR}\right)$ & 38.54 & 38.54 & 38.54 & 38.54 & 38.54 & 38.54 & 38.19 & 38.19 & 37.85 & 37.85 & 37.50 & 37.50 & 37.50 \\
\hline $\begin{array}{l}\text { T2 (NPK Granular 13-6-27 \& } \\
\text { Trichoderma Bio fungicides) }\end{array}$ & 96,53 & 97,92 & 98,26 & 98,31 & 98,96 & 100 & 100 & 100 & 100 & 100 & 100 & 100 & 100 \\
\hline
\end{tabular}

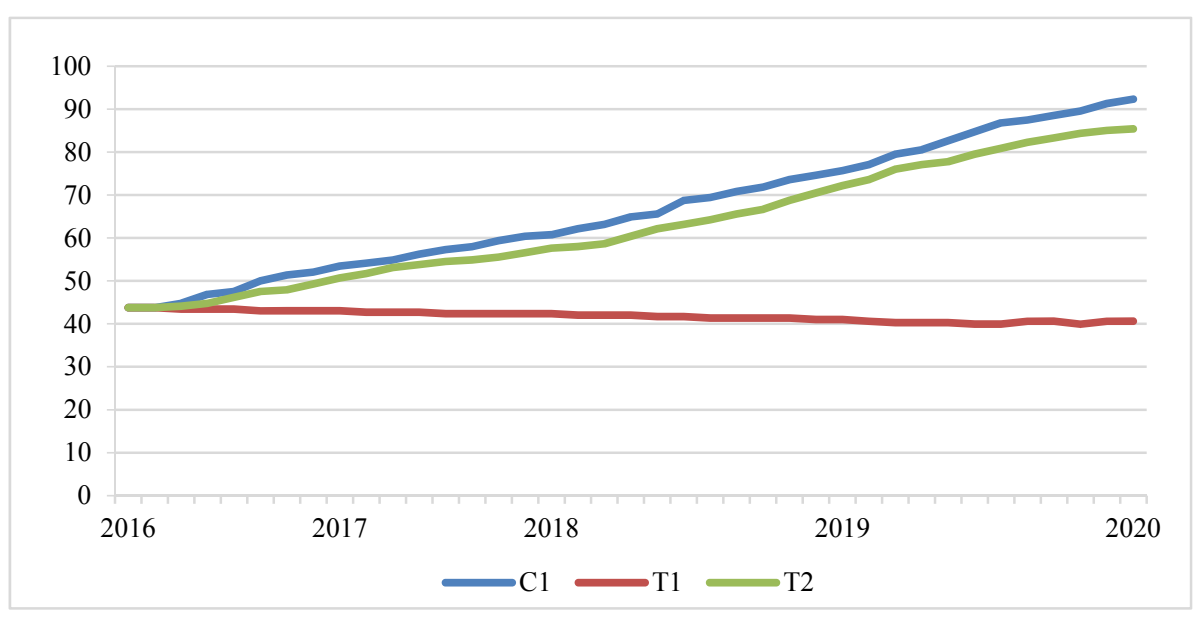

Figure 3. Graph of DSI values from samples on $\mathrm{C} 1, \mathrm{~T} 1$ and $\mathrm{T} 2$ treatment

From Table 3 and Figure 3, can be seen that from observation on C1 (control), T1 (MOAFTM \& CHIPS ${ }^{\circledR}$ ) and T2 (NPK Granular 13-6-27 fertilizer \& Trichoderma bio fungicides) from week 4 (2016) to week 260 (2020). On C1 treatment (control) in week 4 (2016) to week 212 (2020) the DSI value increased from $43.75 \%$ to $100 \%$. On T2 treatment (NPK Granular fertilizer 13-6-27 \& Trichoderma Bio fungicides) the DSI value increased from $43.75 \%$ to $100 \%$ on week 232 (2020). Meanwhile on T1 (MOAFTM \& CHIPS ${ }^{\mathbb{B}}$ ) treatment DSI value decreased from $43.75 \%$ to $37.50 \%$ in weeks 260 (2020). 
When Disease Severity Index value (DSI) increased, it means the severity stage is increased too as seen in C1 (control) treatment and T2 (NPK Granular fertilizer 13-6-27 \& Trichoderma bio fungicides) treatment. Meanwhile DSI value of T1 treatment $\left(\mathrm{MOAF}^{\mathrm{TM}} \& \mathrm{CHIPS}^{\circledR}\right)$ has show significant decrease in the value and have better recovery after the application. The observation is done from 2016 to 2020 .

Based on DSI result on Table 3, to determine if there are differences in effectiveness of each treatment $(\mathrm{Cl}$, T1 and T2) then statistical RAL test was conducted and the result can be seen in Table 4:

Table 4. Differences in each treatment

\begin{tabular}{llll}
\hline (I) Treatment & $(\mathrm{J})$ Treatment & Mean Difference (I-J) & Sig. \\
\hline \multirow{2}{*}{$\mathrm{C} 1$} & $\mathrm{~T} 1$ & $27.47255^{*}$ & .000 \\
\hdashline & $\mathrm{T} 2$ & 4.30380 & .160 \\
$\mathrm{~T} 1$ & $\mathrm{C} 1$ & $-27.47255^{*}$ & .000 \\
\hdashline $\mathrm{T} 1$ & $-24.34281^{*}$ & .000 \\
$\mathrm{~T} 2$ & $\mathrm{C} 1$ & -4.90380 & .160 \\
& $\mathrm{~T} 1$ & $24.34281^{*}$ & .000 \\
\hline
\end{tabular}

Based on Table 4 can be seen differences between C1 (without treatment) and T1 (MOAFTM dan CHIPS ${ }^{\circledR}$ ) shows significant differences, but there are no significant differences shows on T2 treatment (NPK Granular fertilizer 13-6-27 \& Trichoderma Bio fungicides).

On T1 treatment (MOAFTM dan CHIPS $^{\circledR}$ ) shows there are significant differences from comparison to C1 treatment (Control) and T2 (NPK Granular fertilizer 13-6-27 \& Trichoderma Bio fungicides). Furthermore, it can be seen in comparison of T2 treatment (NPK Granular fertilizer 13-6-27 \& Trichoderma Bio fungicides) with $\mathrm{C} 1$ treatment (control) shows no significant differences meanwhile T1 (MOAF ${ }^{\mathrm{TM}}$ dan $\mathrm{CHIPS}^{\circledR}$ ) treatment shows significant differences.

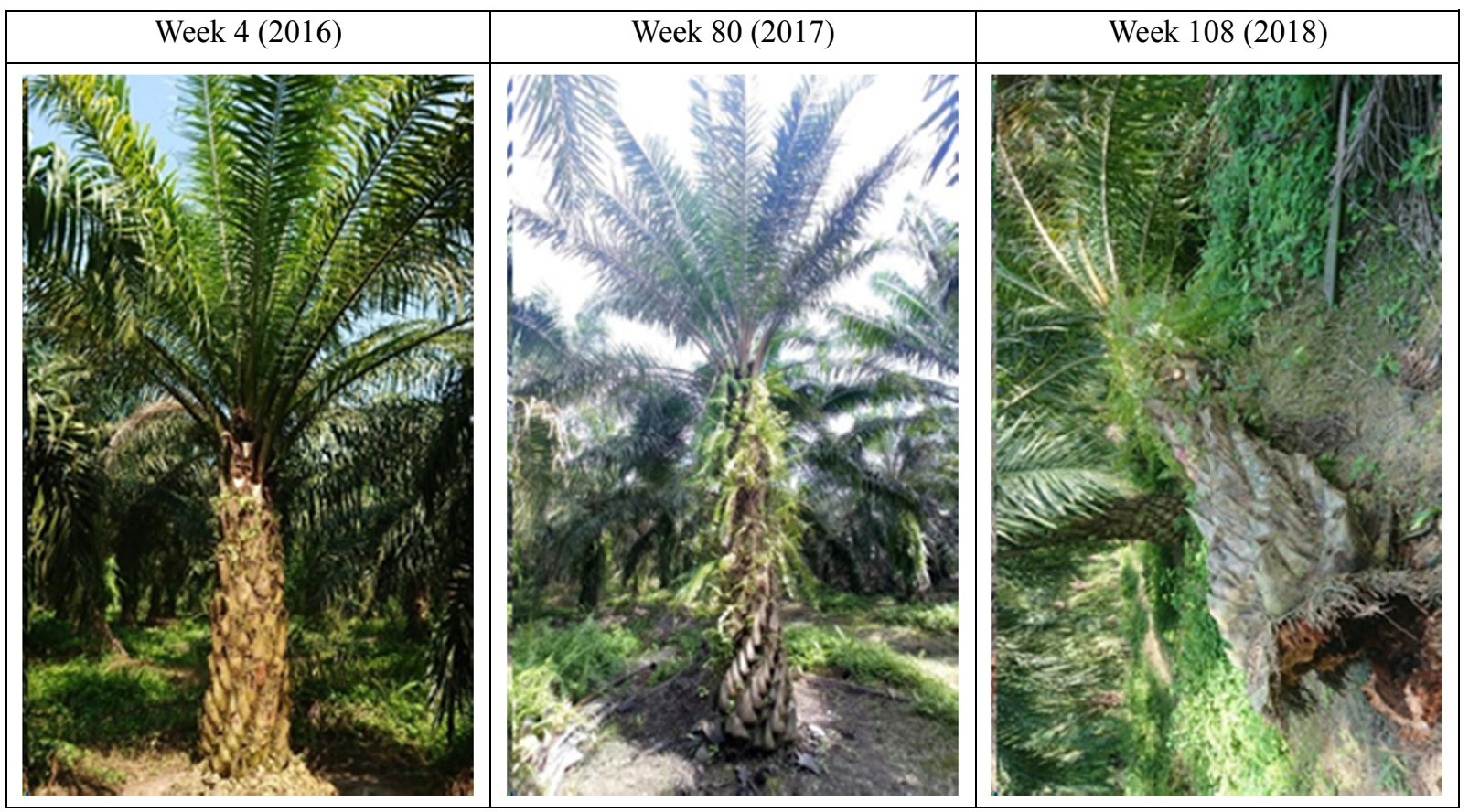

Figure 4. Plant development progress on treatment C1 (control)

From Figure 4 can be seen the plant development progress with $\mathrm{C} 1$ treatment (control) which initially in week 4 (2016) is classified as stage I (yellowish and dull leaf color such as lack of water and flat header surface). On observation in week 80 (2017) the tree can be classified as stage II. In observation in week 108 (2018) the tree that initially stood upright, fell because the tissue inside the tree had been heavily damaged and categorized as 
stage IV. This shows that the $\mathrm{C} 1$ treatment has worsen the disease, from stage I to stage IV as well as the increasing value of the Disease Severity Index during 5 years of observation.
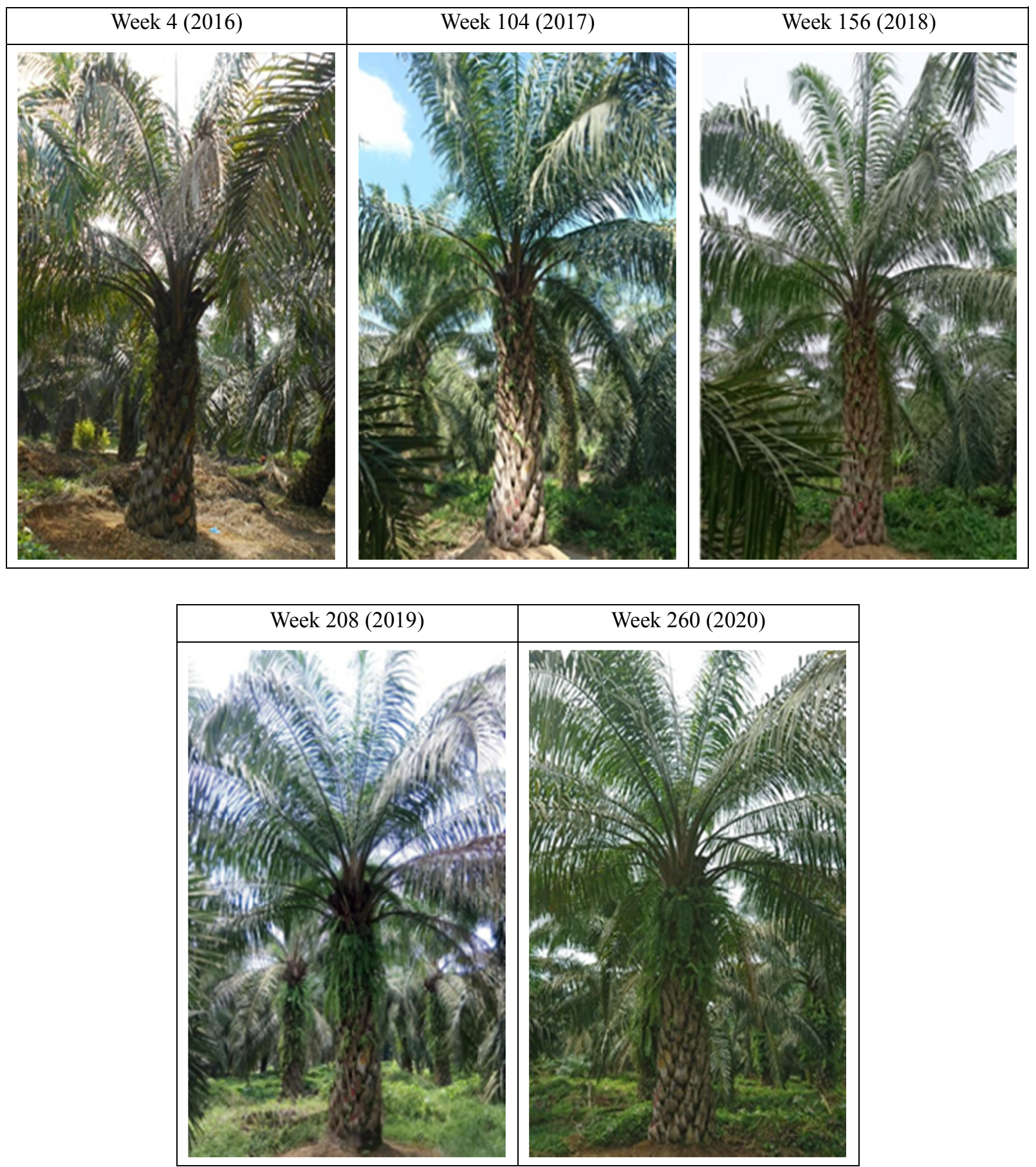

Figure 5. Plant development progress on treatment T1 $\left(\right.$ MOAFTM $^{\text {L }}$ CHIPS $\left.^{\circledR}\right)$

From Figure 5 can be seen the plant development progress with T1 treatment (MOAFTM \& CHIPS $®$ ) which was originally classified in stage II in week 4 (2016) and showing necrosis on the surface of the leaves with the drying lower fronds and two unopened spear leaves. At the 104th week (2017) there were changed of color in leaves and the two spear leaves begin to open up, which indicate that the plant was turning back into stage I. At week 156th (2018) the leaves were growing well and increasing in production every year. Last observation of week 414 (2020) the tree still stands upright. This shows the success of T1 treatment in-field which can be seen 
from the decreasing stage and severity index of the disease during the 5 years of application of MOAF fertilizer ${ }^{\mathrm{TM}}$ and $\mathrm{CHIPS} \otimes$.

Collaboration mechanisms of various microorganisms in CHIPS ${ }^{\circledR}$ suppressed Ganoderma growth rate.

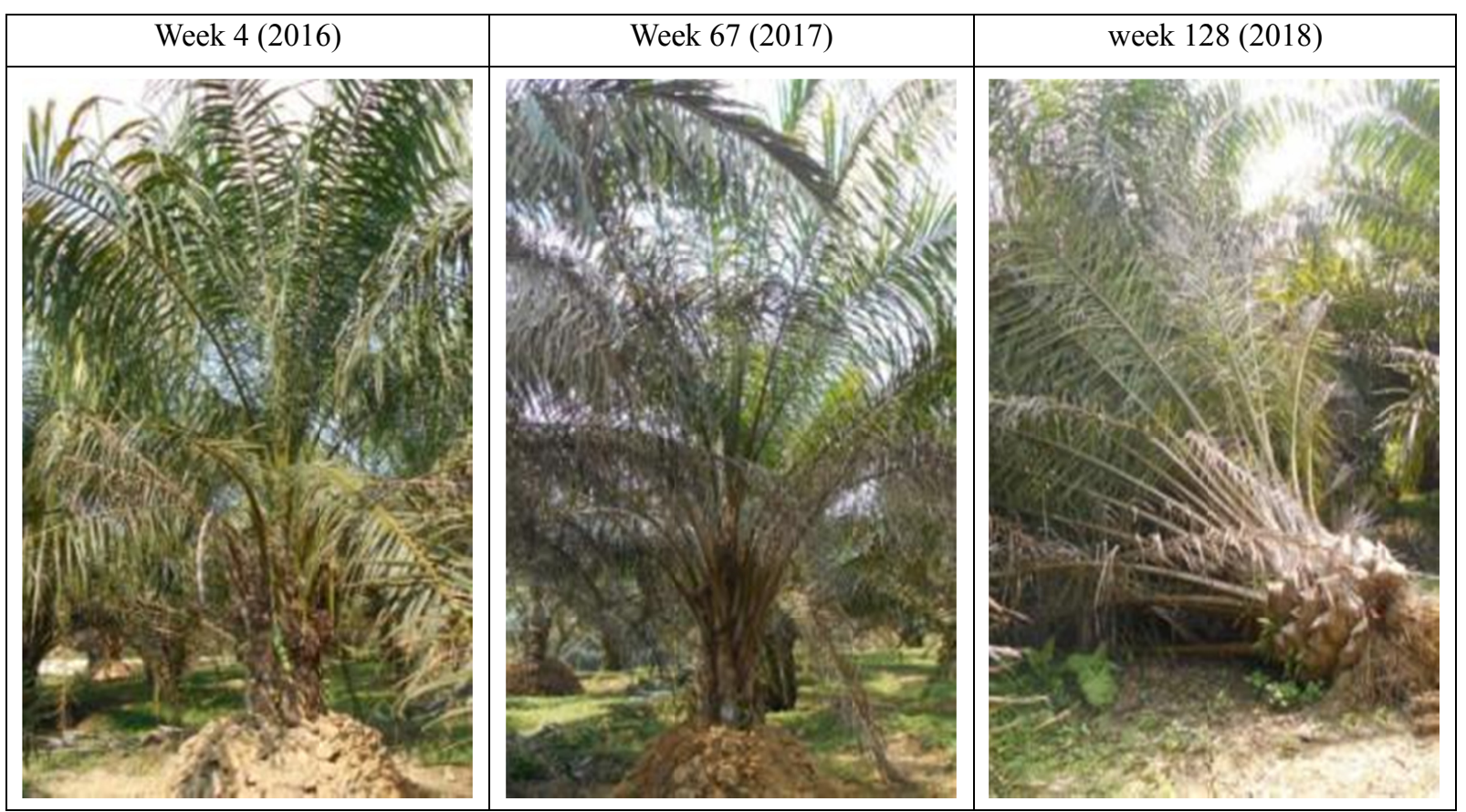

Figure 6. Plant development progress on treatment T2 (NPK Granular fertilizer 13-6-27

\& Trichoderma bio fungicides)

From Figure 6 can be seen that the plant development progress with T2 (NPK Granular Fertilizer 13-6-27 \& Trichoderma bio fungicides) which was originally classified as stage I in week 4th (year 2016). At week 67th (2017) almost all leaves dry,-and three spear leaves were not open, which can be classified as stage III. At week 128th (2018) the condition of the plant worsen, and oil palm fruit production decreased every year. The fallen tree caused by the tissue damaging Ganoderma, could be categorized as stage IV of the disease and the increasing of disease severity index during 5 years of observation with the application of NPK Granular Fertilizer 13-6-27 \& Trichoderma bio fungicides.

By calculating the Revenue to Cost Ratio in eradicating the Ganoderma disease, we can know the profitability comparison among all treatments. Makin et al. (1980) stated that treatment or effort that has the highest R-C ratio shows the most profit.

Table 5. Cost for material and labor cost

\begin{tabular}{|c|c|c|c|}
\hline \multicolumn{2}{|c|}{ Treatment } & \multirow{2}{*}{$\begin{array}{l}\text { Material cost (Rp/kg) } \\
\text { Rp 5,575 }\end{array}$} & \multirow{2}{*}{$\begin{array}{l}\text { Labor Cost (Rp/tree) } \\
\operatorname{Rp} 480\end{array}$} \\
\hline C1 & NPK 13-6-27 & & \\
\hline \multirow{2}{*}{ T1 } & $\mathrm{MOAF}$ & $\operatorname{Rp} 7,200$ & $\operatorname{Rp} 480$ \\
\hline & CHIPS 2,1 & Rp 12,500 & $\operatorname{Rp} 4,500$ \\
\hline \multirow{2}{*}{$\mathbf{T 2}$} & NPK 13-6-27 & Rp 5,575 & $\operatorname{Rp} 480$ \\
\hline & Trichoderma & $\operatorname{Rp} 11,000$ & $\operatorname{Rp} 4,500$ \\
\hline
\end{tabular}


Table 6. Cost for $\mathrm{C} 1$ treatment (Control)

\begin{tabular}{|c|c|c|c|}
\hline \multirow{3}{*}{ Week } & \multicolumn{3}{|c|}{ C1 (Control) } \\
\hline & \multicolumn{3}{|c|}{ NPK 13-6-27 } \\
\hline & Dosage (kg/tree) & Material Cost (Rp/tree) & Labor Cost (Rp/tree) \\
\hline 4 & 2.5 & Rp 13,938 & $\operatorname{Rp} 480$ \\
\hline 20 & 2.5 & Rp 13,938 & $\mathrm{Rp} 480$ \\
\hline \multicolumn{4}{|l|}{32} \\
\hline 40 & 2.5 & Rp 13,938 & $\mathrm{Rp} 480$ \\
\hline 56 & 2.5 & Rp 13,938 & $\mathrm{Rp} 480$ \\
\hline 72 & 2.5 & Rp 13,938 & Rp 480 \\
\hline \multicolumn{4}{|l|}{84} \\
\hline 92 & 2.5 & Rp 13,938 & $\mathrm{Rp} 480$ \\
\hline 108 & 2.5 & Rp 13,938 & $\mathrm{Rp} 480$ \\
\hline 124 & 2.5 & Rp 13,938 & Rp 480 \\
\hline \multicolumn{4}{|l|}{136} \\
\hline 144 & 2.5 & Rp 13,938 & $\mathrm{Rp} 480$ \\
\hline 160 & 2.5 & Rp 13,938 & $\mathrm{Rp} 480$ \\
\hline 176 & 2.5 & Rp 13,938 & $\operatorname{Rp} 480$ \\
\hline \multicolumn{4}{|l|}{188} \\
\hline 194 & 2.5 & Rp 13,938 & $\operatorname{Rp} 480$ \\
\hline 212 & 2.5 & Rp 13,938 & $\operatorname{Rp} 480$ \\
\hline 228 & 2.5 & Rp 13,938 & $\mathrm{Rp} 480$ \\
\hline \multicolumn{4}{|l|}{240} \\
\hline 248 & 2.5 & Rp 13,938 & $\mathrm{Rp} 480$ \\
\hline Total & & Rp 209,063 & $\mathbf{R p} 7, \mathbf{2 0 0}$ \\
\hline Total (1 & & & Rp 216,263 \\
\hline
\end{tabular}

Table 7. Cost for T1 treatment (MOAFTM dan CHIPS $\left.{ }^{\circledR}\right)$

\begin{tabular}{|c|c|c|c|c|c|c|}
\hline \multirow{3}{*}{ Week } & \multicolumn{6}{|c|}{ T1 } \\
\hline & \multicolumn{3}{|c|}{ MOAFTM } & \multicolumn{3}{|c|}{ CHIPS $^{\circledR}$} \\
\hline & Dosage (kg/tree) & Material Cost (Rp) & Labor Cost (Rp) & Dosage (kg/tree) & Material Cost (Rp) & Labor Cost (Rp) \\
\hline 4 & 3 & $\operatorname{Rp} 21,600$ & $\mathrm{Rp} 480$ & 3 & $\operatorname{Rp} 37,500$ & $\operatorname{Rp} 4,500$ \\
\hline \multicolumn{7}{|l|}{20} \\
\hline 32 & 3 & $\operatorname{Rp} 21,600$ & $\mathrm{Rp} 480$ & & & \\
\hline \multicolumn{7}{|l|}{40} \\
\hline 56 & 3 & $\operatorname{Rp} 21,600$ & $\operatorname{Rp} 480$ & & & \\
\hline \multicolumn{7}{|l|}{72} \\
\hline 84 & 3 & Rp 21,600 & $\mathrm{Rp} 480$ & & & \\
\hline \multicolumn{7}{|l|}{92} \\
\hline 108 & 3 & $\operatorname{Rp} 21,600$ & $\mathrm{Rp} 480$ & & & \\
\hline \multicolumn{7}{|l|}{124} \\
\hline 136 & 3 & $\operatorname{Rp} 21,600$ & $\operatorname{Rp} 480$ & 3 & $\operatorname{Rp} 37,500$ & $\operatorname{Rp} 4,500$ \\
\hline \multicolumn{7}{|l|}{144} \\
\hline 160 & 3 & $\operatorname{Rp} 21,600$ & $\mathrm{Rp} 480$ & & & \\
\hline \multicolumn{7}{|l|}{176} \\
\hline 188 & 3 & $\operatorname{Rp} 21,600$ & $\mathrm{Rp} 480$ & & & \\
\hline \multicolumn{7}{|l|}{194} \\
\hline 212 & 3 & $\operatorname{Rp} 21,600$ & $\mathrm{Rp} 480$ & & & \\
\hline \multicolumn{7}{|l|}{228} \\
\hline 240 & 3 & Rp 21,600 & $\mathrm{Rp} 480$ & & & \\
\hline \multicolumn{7}{|l|}{248} \\
\hline Total & & Rp 216,000 & Rp 4,800 & & Rp 75,000 & Rp 9,000 \\
\hline \multicolumn{6}{|c|}{ Total (Material + Labor) } & Rp 304,800 \\
\hline
\end{tabular}


Table 8. Cost for T2 treatment (NPK Granular Fertilizer 13-6-27 \& Trichoderma bio-fungicides)

\begin{tabular}{|c|c|c|c|c|c|c|}
\hline \multirow{3}{*}{ Week } & \multicolumn{6}{|c|}{ T2 } \\
\hline & \multicolumn{3}{|c|}{ NPK 13-6-27 } & \multicolumn{3}{|c|}{ Trichoderma } \\
\hline & Dosage (kg/tree) & Material Cost (Rp) & Labor Cost (Rp) & Dosage (kg/tree) & Material Cost (Rp) & Labor Cost (Rp) \\
\hline$\overline{4}$ & 2.5 & $\operatorname{Rp~13,938}$ & Rp 480 & 0.4 & $\mathrm{Rp} 4,400$ & $\mathrm{Rp} 4,500$ \\
\hline 20 & 2.5 & Rp 13,938 & $\mathrm{Rp} 480$ & & & \\
\hline 32 & & & & 0.4 & $\operatorname{Rp} 4,400$ & $\operatorname{Rp} 4,500$ \\
\hline 40 & 2.5 & Rp 13,938 & $\mathrm{Rp} 480$ & & & \\
\hline 56 & 2.5 & Rp 13,938 & $\mathrm{Rp} 480$ & 0.4 & $\operatorname{Rp} 4,400$ & $\operatorname{Rp} 4,500$ \\
\hline 72 & 2.5 & Rp 13,938 & $\mathrm{Rp} 480$ & & & \\
\hline 84 & & & & 0.4 & $\operatorname{Rp} 4,400$ & $\operatorname{Rp} 4,500$ \\
\hline 92 & 2.5 & $\operatorname{Rp} 13,938$ & $\mathrm{Rp} 480$ & & & \\
\hline 108 & 2.5 & Rp 13,938 & $\mathrm{Rp} 480$ & 0,4 & $\operatorname{Rp} 4,400$ & $\operatorname{Rp} 4,500$ \\
\hline 124 & 2.5 & Rp 13,938 & $\mathrm{Rp} 480$ & & & \\
\hline 136 & & & & 0,4 & $\operatorname{Rp} 4,400$ & $\operatorname{Rp} 4,500$ \\
\hline 144 & 2.5 & Rp 13,938 & $\mathrm{Rp} 480$ & & & \\
\hline 160 & 2.5 & Rp 13,938 & $\mathrm{Rp} 480$ & 0,4 & $\operatorname{Rp} 4,400$ & $\operatorname{Rp} 4,500$ \\
\hline 176 & 2.5 & Rp 13,938 & Rp 480 & & & \\
\hline 188 & & & & 0,4 & $\operatorname{Rp} 4,400$ & $\operatorname{Rp} 4,500$ \\
\hline 194 & 2.5 & Rp 13,938 & $\mathrm{Rp} 480$ & & & \\
\hline 212 & 2.5 & Rp 13,938 & Rp 480 & 0,4 & $\operatorname{Rp} 4,400$ & $\operatorname{Rp} 4,500$ \\
\hline 228 & 2.5 & Rp 13,938 & Rp 480 & & & \\
\hline 240 & & & & 0,4 & $\operatorname{Rp} 4,400$ & $\operatorname{Rp} 4,500$ \\
\hline 248 & 2.5 & Rp 13,938 & $\mathrm{Rp} 480$ & & & \\
\hline Total & & Rp 209,063 & $\operatorname{Rp} 7,200$ & & Rp 44,000 & Rp 45,000 \\
\hline Total ( & terial + labor) & & & & & Rp 305,263 \\
\hline
\end{tabular}

Table 9. Comparison between cost for treatment $\mathrm{C} 1, \mathrm{~T} 1$ and $\mathrm{T} 2$ for 5 years

\begin{tabular}{|c|c|c|c|c|}
\hline \multirow{2}{*}{ Year } & & \multicolumn{3}{|c|}{ Material Cost + Labor Cost } \\
\hline & & C1 (NPK 13-6-27) & T1 $\left(\right.$ MOAF $^{\mathrm{TM}}+$ CHIPS $\left.^{\circledR}\right)$ & T2 (NPK + Trichoderma) \\
\hline \multirow{2}{*}{2016} & SM I & $\operatorname{Rp} 2,076,120$ & $\operatorname{Rp} 4,613,760$ & $\operatorname{Rp} 2,716,920$ \\
\hline & SM II & Rp 980,390 & Rp $1,589,760$ & Rp $1,664,443$ \\
\hline \multirow{2}{*}{2017} & SM I & Rp $1,874,275$ & Rp $1,589,760$ & $\operatorname{Rp} 2,537,145$ \\
\hline & SM II & Rp 879,468 & Rp $1,589,760$ & $\mathrm{Rp} 1,501,220$ \\
\hline \multirow{2}{*}{2018} & SM I & Rp $1,715,683$ & Rp $1,589,760$ & $\operatorname{Rp} 2,287,418$ \\
\hline & SM II & $\mathrm{Rp} 778,545$ & $\operatorname{Rp} 4,613,760$ & Rp 1,337,998 \\
\hline \multirow{2}{*}{2019} & SM I & Rp $1,427,332$ & Rp $1,589,760$ & $\operatorname{Rp} 2,069,908$ \\
\hline & SM II & Rp 663,205 & Rp $1,589,760$ & $\mathrm{Rp} 1,212,510$ \\
\hline \multirow{2}{*}{2020} & SM I & Rp $1,239,905$ & Rp $1,589,760$ & $\operatorname{Rp} 1,820,180$ \\
\hline & SM II & Rp 576,700 & Rp $1,589,760$ & Rp 1,058,188 \\
\hline \multicolumn{2}{|c|}{ Total (Rp) } & Rp 12,211,623 & Rp 21,945,600 & Rp 18,205,930 \\
\hline
\end{tabular}


Table 10. Comparison income between treatment $\mathrm{C} 1, \mathrm{~T} 1$ and $\mathrm{T} 2$ for 5 years

\begin{tabular}{|c|c|c|c|c|}
\hline \multirow{2}{*}{ Year } & & \multicolumn{3}{|c|}{ Production (kg/Semester) } \\
\hline & & C1 (NPK 13-6-27) & T1 $\left(\right.$ MOAF $^{\mathrm{TM}}+$ CHIPS $\left.^{\circledR}\right)$ & T2 (NPK + Trichoderma) \\
\hline \multirow{2}{*}{2016} & SM I & 2,843 & 2,812 & 2,873 \\
\hline & SM II & 3,756 & 3,884 & 3,899 \\
\hline \multirow{2}{*}{2017} & SM I & 2,484 & 3,661 & 3,129 \\
\hline & SM II & 2,921 & 4,475 & 3,669 \\
\hline \multirow{2}{*}{2018} & SM I & 2,019 & 4,439 & 3,109 \\
\hline & SM II & 2,281 & 5,425 & 3,600 \\
\hline \multirow{2}{*}{2019} & SM I & 1,368 & 5,216 & 3,048 \\
\hline & SM II & 1,554 & 6,376 & 3,564 \\
\hline \multirow{2}{*}{2020} & SM I & 899 & 5,994 & 3,006 \\
\hline & SM II & 1,035 & 7,326 & 3,434 \\
\hline \multicolumn{2}{|c|}{ Total Production (kg/5 years) } & 21,160 & 49,608 & 33,331 \\
\hline \multicolumn{2}{|c|}{ Assumsion FFB price $(\mathrm{Rp} / \mathrm{kg})$} & Rp 1,500 & 1,500 & 1,500 \\
\hline \multicolumn{2}{|c|}{ Total Income (Rp) } & $\mathbf{R p ~ 3 1 , 7 4 0 , 0 0 0}$ & Rp 74,412,000 & Rp 49,996,500 \\
\hline
\end{tabular}

Table 11. Comparison between Revenue, Cost, and Profit for treatment C1, T1, and T2 for 5 years

\begin{tabular}{|c|c|c|c|}
\hline Variabel & C1 (NPK 13-6-27) & T1 $\left(\right.$ MOAF $^{\mathrm{TM}}+$ CHIPS $\left.^{(\mathbb{R}}\right)$ & T2 (NPK + Trichoderma) \\
\hline Revenue & $\operatorname{Rp} 31,740,000$ & $\operatorname{Rp} 74,412,000$ & $\operatorname{Rp} 49,996,500$ \\
\hline Cost & Rp 12,211,623 & $\operatorname{Rp} 21,945,600$ & Rp $18,205,930$ \\
\hline Profit & Rp 19,528,377 & Rp 52,466,400 & Rp 31,790,570 \\
\hline $\mathrm{R} / \mathrm{C}$ Ratio & 2,59 & 3,39 & 2,74 \\
\hline
\end{tabular}

In Table 11, the total cost incurred for $\mathrm{C} 1$ treatment (control) in five years of observation amounted to $\mathrm{Rp}$ $12,211,623$ and the total revenue obtained was $\mathrm{Rp} 31,740,000$ so that the profit obtained is Rp. 19,528,377. In T1 treatment (MOAFTM \& CHIPS ${ }^{\circledR}$ ), the total incurred in five years of observation is Rp 21,945,600 and total revenue

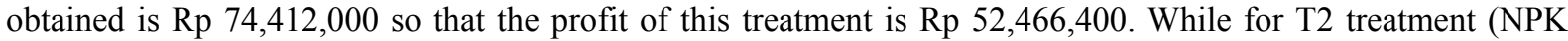

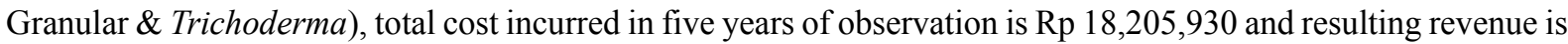
Rp 49,996,500 so that the profit obtained is Rp 31,790,570. T1 treatment (MOAFTM \& CHIPS ${ }^{\circledR}$ ) has the greatest profit on this observation.

Among all treatments for $\mathrm{C} 1, \mathrm{~T} 1$, and $\mathrm{T} 2$, it can be known that the highest $\mathrm{R}-\mathrm{C}$ ratio value is in $\mathrm{T} 1$ treatment (MOAFTM \& CHIPS ${ }^{\mathrm{T}}$ ) amounting to 3.39 which is the most profitable treatment.

\section{Conclusion}

T1 treatment applied with MOAFTM \& CHIPS ${ }^{\circledR}$ is the most effective treatment that can be seen from decreased DSI value from $43.75 \%$ to $37.50 \%$ and shows better recovery than other treatments. Proliferation of fungal biocontrol agents in CHIPS ${ }^{\circledR}$ affects the success of Ganoderma suppression in the field. For T2 treatment by using NPK Granular Fertilizer 13-6-27 \& Trichoderma bio fungicides which has increased the value of DSI from $43.75 \%$ to $100 \%$ in week 232 (2020). Treatment C1 (control) has the highest increase DSI value that reach $100 \%$ in week 212 (2020). If the DSI value is higher, the possibility of fall and dead trees is higher too. This experiment shows that $\mathrm{T} 1$ treatment (MOAFTM and CHIPS ${ }^{\circledR}$ ) showed significant difference and successful treatment compared to other treatments and had the highest profit or Revenue to Cost Ratio of 3.39.

\section{References}

Boulord, R., Carasco-Lacombe, C., Klopp, C., Lee, Y., Tan, J., Syed, S. S. R., ... Fisher, M. C. (2017). About Ganoderma boninense in oil palm plantations of Sumatra and peninsular Malaysia: Ancient population expansion, extensive gene flow and large scale dispersion ability. Fungal Biology, 121(6-7), 529-540. https://doi.org/10.1016/j.funbio.2017.01.001

Cooper, R. M. (2021). Exported Abstract record(s) (pp. 1-2).

Jing, C. J. (2007). Kepatogenan Ganoderma Boninense Pada Kelapa Sawit Dan Hubungan Biologinya Dengan Ganoderma spp. dari pada Perumah Palma Lain. Pusat Pengajian Sains Patologi Tumbuhan, Malaysia. Procedia Manufacturing, 30(June), 588-595. 
Liza, I., Walter, A. W. C. Y., Faizal, M., Bakar, A., Idris, A. S., Diba, F., .. Murad, A. (2018). Data in Brief Transcriptome datasets of oil palm pathogen Ganoderma boninense. Data in Brief, 17, 1108-1111. https://doi.org/10.1016/j.dib.2018.02.027

Mohd, Z., \& Faridah, A. (2008). Disease Suppression in Ganoderma-infected Oil Palm Seedlings Treated with Trichoderma harzianum. Plant Protection Science, 44(3), 101-107.

Paterson, R. R. M. (2007). Ganoderma disease of oil palm-A white rot perspective necessary for integrated control. Crop Protection, 26(9), 1369-1376. https://doi.org/10.1016/j.cropro.2006.11.009

Priwiratama, H., \& Susanto, A. (2014). Utilization of Fungi for the Biological Control of Insect Pests and Ganoderma Disease in the Indonesian Oil Palm Industry. Agricultural Science and Technology: A, 2014(2), 103-111.

Priwiratama, H., Prasetyo, A., \& Susanto, A. (2014). Pengendalian Penyakit Busuk Pangkal Batang Kelapa Sawit secara Kultur Teknis. Jurnal Fitopatologi Indonesia, 10(1), 1-7. https://doi.org/10.14692/jfi.10.1.1

Sundram, S., Abdullah, F., Ahmad, Z. A. M., \& Yusuf, U. K. (2008). Efficacy of single and mixed treatments of trichoderma harzianum as biocontrol agents of ganoderma basal stem rot in oil palm. Journal of Oil Palm Research, 20(June), 470-483.

Susanto, A., Prasetyo, A., \& Wening, S. (2013). Laju Infeksi Ganoderma pada Empat Kelas Tekstur Tanah. Jurnal Fitopatologi Indonesia, 9(2), 39-46. https://doi.org/10.14692/jfi.9.2.39

\section{Copyrights}

Copyright for this article is retained by the author(s), with first publication rights granted to the journal.

This is an open-access article distributed under the terms and conditions of the Creative Commons Attribution license (http://creativecommons.org/licenses/by/4.0/). 